\title{
Radiation induces autophagic cell death via the p53/DRAM signaling pathway in breast cancer cells
}

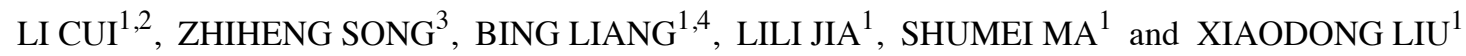 \\ ${ }^{1}$ Key Laboratory of Radiobiology (Ministry of Health), School of Public Health, Jilin University, Changchun, Jilin 130021; \\ ${ }^{2}$ Changchun Central Hospital, Changchun, Jilin 130051; ${ }^{3}$ Jilin Province People's Hospital, Changchun, Jilin 130021; \\ ${ }^{4}$ Department of Obstetrics and Gynecology Care, School of Nursing, Jilin University, Changchun, Jilin 130021, P.R. China
}

Received November 30, 2015; Accepted March 15, 2016

DOI: $10.3892 /$ or.2016.4752

\begin{abstract}
Autophagy is known to play a role in the response of breast cancer cells to radiation therapy. However, the mechanisms that mediate the process of autophagy and contribute to radiation-induced cell death and cell survival remain to be fully characterized. Therefore, in this study, the functional role of autophagy in radiation-induced cytotoxicity in breast cancer cells was investigated. After MCF-7 cells were exposed to various doses of radiation, increased monodansylcadaverine (MDC) staining and a greater deposition of LC3-positive puncta were observed. Expression of the autophagy-related proteins, Beclin 1 and LC3-II, were also found to be upregulated. Radiation-induced autophagic cell death was partially abrogated following the administration of 3-methyladenine (3-MA) and in knockdown experiments of Atg5 and Beclin 1. In the gene microarray analysis performed after irradiation, a number of differentially expressed genes were identified. In particular, upregulation of both the mRNA and protein levels of the autophagy-related genes, DRAM and TIGAR, were detected. However, inhibition of autophagy by 3-MA reduced the radiation-induced upregulation of LC3-II and DRAM. Conversely, silencing of p53 downregulated the expression of LC3-II and DRAM following radiation. Silencing of DRAM reversed the upregulation of LC3-II and DRAM following radiation, partially blocked radiation-induced cell death, and no significant change in p53 expression was detected. Based on these results, the p53/DRAM signaling pathway appears to contribute to radiation-induced autophagic cell death in MCF-7 breast cancer cells.
\end{abstract}

Correspondence to: Dr Xiaodong Liu or Dr Shumei Ma, Key Laboratory of Radiobiology (Ministry of Health), School of Public Health, Jilin University, 1163 Xinmin Street, Changchun, Jilin 130021, P.R. China

E-mail: 821996371@qq.com

E-mail: 827229953@qq.com

Key words: DRAM, p53, autophagy, radiation, breast cancer cells

\section{Introduction}

Autophagy is a highly conserved metabolic pathway that is required for intracellular degradation of long-lived proteins or damaged organelles. Initially, the autophagic process includes the formation of double-membraned autophagosomes, and these subsequently fuse with lysosomes to degrade the contents inside $(1,2)$. In cancer cells, autophagy clears damaged organelles and unfolded proteins, and also provides cellular energy to enhance cell survival; while chronic or excessive autophagy can contribute to cell death (3).

Currently, breast cancer is the most prevalent cancer diagnosed in women, with an estimated 1.8 million cases reported worldwide in 2013 (4). Radiation is commonly adopted as an adjuvant therapy for the management of breast cancer (5). However, there is growing evidence that autophagy is induced by ionizing radiation, and this induction plays a crucial role in radiosensitivity $(6,7)$. Furthermore, the regulatory effect of autophagy in radiation-induced cell death remains controversial, and the underlying molecular mechanisms remain to be fully characterized.

Several autophagy-related genes (Atgs) have been identified in relation to the autophagy machinery. For example, Beclin 1 (the mammalian orthologue of yeast Atg6) and Atg5 are required for the biogenesis of autophagosomes (8). The autophagy pathway is also associated with multiple intracellular signaling pathways. In particular, damage-regulated autophagy modulator (DRAM), a p53 signaling effector, is a lysosomal protein that contributes to p53-regulated autophagy induction (9). The findings of the present study suggest that autophagy is induced by DRAM which leads to increased expression of beclin 1 and production of p53 (10). In addition, p53 has been shown to have a critical role in inducing DRAM-mediated autophagy in normal hepatocytes (7702) and hepatocellular carcinoma HepG2 cells in response to starvation, while p53 overexpression induces DRAM-mediated autophagy (11). Recent evidence also indicates that the p53/DRAM-autophagy axis contributes to anticancer reagents that induce cytotoxicity in breast cancer cells $(12,13)$. However, the potential involvement of the p53/DRAM signaling pathway in radiation-induced autophagic cell death remains unknown.

In this study, activation of autophagy in MCF-7 breast cancer cells was investigated following ionizing radiation 
treatment. Various assays were performed to characterize the radiation-induced autophagy that was achieved. In particular, the gene, $D R A M$, was identified in a gene microarray analysis, thereby recognizing a potential role for this gene in radiation-induced autophagic cell death.

\section{Materials and methods}

Reagents. The following reagents were purchased as indicated: Dulbecco's modified Eagle's medium (DMEM) culture medium and fetal bovine serum (FBS) (Life Technologies, Grand Island, NY, USA); trypan blue solution (Sigma-Aldrich, St. Louis, MO, USA); protease inhibitor cocktail (Roche Diagnostics, Basel, Switzerland); siRNAs targeting Atg5 (sc-41445) and Beclin 1 (sc-29797) and control siRNA-A (sc-37007) (Santa Cruz Biotechnology, Santa Cruz, CA, USA); anti-Atg5 (\#2630) and anti-Beclin 1 (\#3738) primary antibodies (Cell Signaling Technology, Beverly, MA, USA); mouse monoclonal anti-p53 antibody (sc-126; Santa Cruz Biotechnology); rabbit monoclonal anti-TIGAR (ab37910) and anti-DRAM (ab68987) antibodies (Abcam, Cambridge, UK); rabbit antiLC3 (no. 12741P) antibody (Cell Signaling Technology); mouse anti-GAPDH (no. A3853) and anti-actin (no. A5441) primary antibodies (Sigma-Aldrich); horseradish peroxidase (HRP)conjugated goat anti-rabbit $\operatorname{IgG}(\mathrm{H}+\mathrm{L}$, no. 170-6515) and goat anti-mouse IgG (H+L, no. 170-6516) antibodies (Bio-Rad Laboratories, Hercules, CA, USA); and primers used for PCR were synthesized by Takara (Dalian, China).

Cell culture and radiation. MCF-7 breast cancer cells were maintained in DMEM supplemented with 5\% FBS, $100 \mathrm{U} / \mathrm{ml}$ of penicillin, and $100 \mu \mathrm{g} / \mathrm{ml}$ of streptomycin in a humidified $5 \% \mathrm{CO}_{2}$ incubator at $37^{\circ} \mathrm{C}$. The cells were exposed to $2,4,6$, or 8 Gy radiation at a rate of $0.40 \mathrm{~Gy} / \mathrm{min}$ using an X-ray generator (X-RAD 320ix; Precision X-Ray Inc., North Branford, CT, USA). Untreated cells were used as a control.

Monodansylcadaverine (MDC) staining assay. MDC staining was used to determine the presence of autophagic vacuoles. Briefly, MCF-7 cells were pre-seeded onto glass cover slips and $24 \mathrm{~h}$ later the cells were irradiated. After an additional $24 \mathrm{~h}$, the irradiated cells were washed twice with cold phosphate-buffered saline (PBS) and then were subsequently incubated with $0.05 \mathrm{mM}$ MDC solution in DMEM for $1 \mathrm{~h}$ at $37^{\circ} \mathrm{C}$. After three washes with PBS, the cells were fixed in $4 \%$ paraformaldehyde for $15 \mathrm{~min}$ and then were examined with a confocal scanning microscope (OLYMPUS-FA500; Olympus, Japan). Fluorescence intensity was quantified by using a FACSCanto flow cytometer (BD Biosciences, Franklin Lakes, NJ, USA).

CCK-8 assay for cell viability. Cells were seeded in 96-well plates $\left(5 \times 10^{3}\right.$ cells/well) and were maintained in complete culture medium for $24 \mathrm{~h}$. Forty-eight hours after treatment, CCK-8 (Dojindo) solution was added to each well (10 $\mu \mathrm{l})$. After $2 \mathrm{~h}$, absorbance values for each plate were measured at $450 \mathrm{~nm}$ using a microplate reader (Synergy HT; Bio-Tek, Winooski, VT, USA).

Plasmid transfection. A pcDNA3.1-RFP-LC3 (or RFP-LC3) plasmid expressing the autophagy-related gene, $l c 3$, was constructed by inserting the $l c 3$ cassette into the EcoRI and BamHI sites of the pcDNA3.1-RFP vector (VPY0003; Changsha YRBio, Hunan, China). Following sequencing confirmation of the resulting ligated vector, MCF-7 cells were transfected with RFP-LC3 using Lipofectamine 2000 reagent (Invitrogen, Carlsbad, CA, USA) as described previously $(14,15)$. For each transfection, 30 fields were randomly selected and the number of cells expressing RFP-labeled LC3 puncta were calculated in each field. Three independent experiments were conducted for each group.

Transfection of small interfering RNA (siRNA). To knockdown expression levels of Atg5 and Beclin 1, MCF-7 cells were transfected with siRNAs targeting Atg5 and Beclin 1, as described in our previous study (16). In addition, a scrambled siRNA was used as a control. Briefly, one day prior to transfection, MCF-7 cells were seeded into $100-\mathrm{mm}$ tissue culture plates. Forty-eight hours later when the cells reached 30-50\% confluency, the cells were transfected with each of the three types of siRNAs (siAtg5, siBeclin1 and sicon, respectively) diluted 1:5 with Oligofectamine reagent (Invitrogen) and then diluted with $40 \mu \mathrm{l}$ of serum-free DMEM. After 5-10 min at room temperature (RT), another tube containing $10 \mu \mathrm{l}$ of $20 \mu \mathrm{M}$ siRNA added into $440 \mu \mathrm{l}$ of serum-free DMEM was added to each diluted Oligofectamine mixture. After 15-20 min at RT, the siRNA-Oligofectamine-reagent complex solution was added to $2.5 \mathrm{ml}$ of serum-free DMEM and this mixture was added to each dish of cells that had been washed once with serum-free DMEM. The final concentration of each siRNA in medium was $40 \mathrm{nM}$. As a control, $2.5 \mathrm{ml}$ serum-free DMEM with only the sicon siRNA was added onto the cells. After the transfected cells were incubated for $4 \mathrm{~h}$ at $37^{\circ} \mathrm{C}$ in a $5 \% \mathrm{CO}_{2}$ incubator, $2.5 \mathrm{ml}$ serum-free DMEM and $400 \mu \mathrm{l}$ serum (FBS) were added to each plate. On the fourth day after transfection, $2 \times 10^{6}$ cells from each transfection were divided equally into the wells of a 6 -well plate. On the sixth or seventh day following transfection, each set of cells was harvested for analysis.

Western blot analysis. Total protein extracts were collected from MCF-7 cells in RIPA lysis buffer [HEPES (50 mM), $\mathrm{NaCl}(150 \mathrm{mM})$, EDTA (1 mM), EGTA (2.5 mM), NaF (10 mM), DTT $(1 \mathrm{mM})$, sodium orthovanadate $(1 \mathrm{mM})$, PMSF (1 mM), NP-40 (1\%), and SDS (0.1\%)]. Proteins were heated to $95^{\circ} \mathrm{C}$ for $5 \mathrm{~min}$ and $40 \mu \mathrm{g}$ of the extracted proteins was prepared in 2-ml aliquots and were mixed with $20 \mu \mathrm{l}$ of protease inhibitor cocktail. After a 5-min incubation on ice, the samples were sonicated and centrifuged at 12,000 rpm for $10 \mathrm{~min}$. The resulting supernatants were transferred to new tubes, and each were mixed with $5 \mathrm{X}$ SDS before being loaded onto a $10 \%$ SDS-PAGE gel. Following transfer of the gels to nitrocellulose membranes, the membranes were blocked with 5\% non-fat dried milk in Tris-buffered saline (TBS) containing $10 \mathrm{mM}$ Tris ( $\mathrm{pH} 7.5$ ), $100 \mathrm{mM} \mathrm{NaCl}$, and $0.1 \%$ Tween-20 at RT. After $1.5 \mathrm{~h}$, the membranes were incubated with the appropriate primary antibodies overnight at $4^{\circ} \mathrm{C}$. The dilutions used for the primary antibodies were: anti-Beclin 1 $(1: 1,000)$, anti-LC3 (1:1,000), anti-GAPDH $(1: 1,000)$, anti-Atg5 $(1: 1,000)$, anti-actin $(1: 1,000)$, anti-p53 (1:500), anti-TIGAR $(1: 1,000)$ and anti-DRAM $(1: 300)$. After the membranes were 
washed, the membranes were incubated with the appropriate HRP-conjugated secondary antibodies at RT. After $1 \mathrm{~h}$, bound antibodies were visualized with a chemiluminescence detection system according to the manufacturer's instructions (Pierce, Burlingame, CA, USA). Detection of GAPDH and actin were used as loading controls.

Determination of cell death and apoptosis. MCF-7 cells were plated onto 6-well plates and were pre-incubated with or without the autophagy inhibitor, 3-methyladenine (3-MA), for $1 \mathrm{~h}$ followed by exposure to $4-\mathrm{Gy}$ radiation. At the indicated time-points after irradiation, the cells were collected and washed with PBS three times.

To evaluate cell death, cells were stained with trypan blue dye which is excluded from live cells yet penetrates into dead cells and produces a red fluorescent signal that can be quantified by flow cytometry (FACSCanto; BD Biosciences).

To detect cell apoptosis, collected cells were stained with an Annexin V-FITC Apoptosis Detection kit I according to the manufacturer's recommendation (BD Biosciences). After the cells were counted by flow cytometry (FACSCanto; BD Biosciences), they were analyzed with FCS Express v2.0 software (De Novo Software, Thornhill, ON, Canada).

Short hairpin RNA (shRNA) constructs and transfection. shRNAs were designed according to 'www.idtdna.com' and were synthesized, denatured, annealed, and ligated to the pSUPER vector within the BglII and HindIII sites. The shRNA sequences for targeting DRAM were: sense, 5'-AGCTTGCCA CATACGGATGGTCATTTCAAGAGAATGACCATCCGTA TGTGGCTTTTTA-3' and antisense, 5'-GATCTAAAAAGC CACATACGGATGGTCATTCTCTTGAAATGACCATCCG TATGTGGCA-3'. The shRNA sequences for targeting p53 were: sense, 5'-GATCCCCGGAGGTTGTGAGGCACTGCTT CAAGAGAGCAGTGCCTCACAACCTCCTTTTTA-3' and antisense, 5'-AGCTTAAAAAGGAGGTTGTGAGGCACTG CTCTCTTGAAGCAGTGCCTCACAACCTCCGGG-3'. A pSUPER construct expressing a scrambled sequence with no significant homology to any known mammalian mRNA was used as a control. All of the plasmids were constructed in our laboratory. The plasmids were transfected into 293T packaging cells by calcium phosphate co-precipitation (Ampho Pack plasmid $10 \mu \mathrm{g}$, pSUPER-shRNA plasmid $10 \mu \mathrm{g}, 2 \mathrm{M} \mathrm{CaCl}_{2}$ $31 \mu \mathrm{l}, \mathrm{ddH}_{2} \mathrm{O}$ to $250 \mu \mathrm{l}$, and $250 \mu \mathrm{l} 2 \mathrm{X}$ HEPES buffer salt solution) and supernatants containing pseudovirus particles were collected after $72 \mathrm{~h}$ and applied to MCF-7 cells in the presence of Polybrene $(8 \mu \mathrm{g} / \mathrm{ml})$. Stable cell clones were selected in the presence of puromycin $(0.8 \mu \mathrm{g} / \mathrm{ml})$ for 7 days.

Gene microarray analysis. MCF-7 cells were exposed to 0, 4, and $8 \mathrm{~Gy}$ of radiation for $4 \mathrm{~h}$ and then total RNA samples were collected using an mRNA isolation kit (Ambion, Austin, TX, USA). All of the RNA samples were labeled with Cy5/Cy3 and then were hybridized to a Human Whole Genome OneArray (Phalanx Biotech Group, Taiwan). The hybridized chips were scanned with an Axon 4000 scanner (Molecular Devices, Sunnyvale, CA, USA) and spot quantification was performed by using GenePix 4.1 software (Molecular Devices). Hierarchical clustering was performed by Cluster 3.0 software (Molecular Devices) for the expression profiles obtained. Differentially expressed genes that exhibited greater than a 2-fold change were further analyzed by Pathway-Express software (OntoTools, Wayne State University, Detroit, MI, USA).

Quantitative real-time PCR ( $q P C R$ ). Total RNA was isolated using RNAiso Plus reagent according to the manufacturer's instructions (Takara). The quality and quantity of the RNA samples collected were analyzed by measuring the A260/A280 ratio for each with an ultraviolet spectrophotometer (Beckman Coulter, Miami, FL, USA). Each RNA sample (2 $\mu \mathrm{g})$ was subjected to reverse transcription using a PrimeScript RT reagent kit (Takara). All of the primers were designed with Primer5.0 (Premier, Canada) and their specificity was verified by Blast NCBI. The forward and reverse primer sequences for the target genes included: 5'-CAAGTGTGGGCTGCTGA GGA-3' and 5'-AGCCTGGGTACAGGTTGTTGATG-3' for DRAM; 5'-CAGTGATCTCATGAGGACAAAGCA-3' and 5'-CCATGGCCCTCAGCTCACTTA-3 for TIGER; and 5'-GCACCGTCAAGGCTGAGAAC-3' and 5'-TGGTGAAGA CGCCAGTGGA-3' for GAPDH, respectively in each case. To perform qPCR (Stratagene MX3000P; Agilent Technologies, Santa Clara, CA, USA), the SYBR Premix Ex TaqII reagent (Takara) was used to amplify the resulting cDNAs. The conditions for the qPCR cycles included: an activation step at $95^{\circ} \mathrm{C}$ for $10 \mathrm{sec}, 40$ cycles of denaturation at $95^{\circ} \mathrm{C}$ for $20 \mathrm{sec}$, and an annealing step at $60^{\circ} \mathrm{C}$ for $20 \mathrm{sec}$. The formula, $2^{-\Delta \Delta \mathrm{CT}}$, where $\Delta \mathrm{CT}$ is the value from the threshold cycle (CT) of the treated sample subtracted from the CT value of the untreated or zero time-point control sample was used to calculate mRNA levels (17). The relative amount of mRNA was normalized to the levels of GAPDH mRNA.

Statistical analysis. All of the experiments described were performed in triplicate. For the analysis of cell death, each experiment was performed with 3-6 replicates. Data are presented as the mean \pm standard deviation (SD). Student's t-test was performed for statistical analyses and a p-value $<0.05$ was considered statistically significant.

\section{Results}

Radiation induces autophagy in breast cancer cells. MDC is a lysosomotropic compound that is commonly used for the detection of autophagic vacuoles in cells (18). Thus, staining with MDC was initially performed to investigate the induction of autophagy in MCF-7 breast cancer cells exposed to 4 Gy of radiation. Twenty-four hours later, a higher number of MDC-labeled vacuoles was observed in the irradiated cells than that detected in the control cells (Fig. 1A). Moreover, a dose-dependent increase in the number of positively-stained cells was observed within $24 \mathrm{~h}$ after irradiation. RFP-LC3 is a fluorescent protein which labels both autophagosomes and autolysosomes (19), and a higher number of LC3-labeled puncta were also observed in the irradiated cells compared with the control cells (Fig. 1A). In addition, the percentage of cells expressing LC3-positive puncta gradually increased with time after irradiation (Fig. 1C). In accordance with these results, western blot analysis demonstrated that expression of the autophagy-related proteins, Beclin 1 and LC3-II, were upregulated $24 \mathrm{~h}$ after the exposure of the MCF-7 cells to 4-Gy 
A
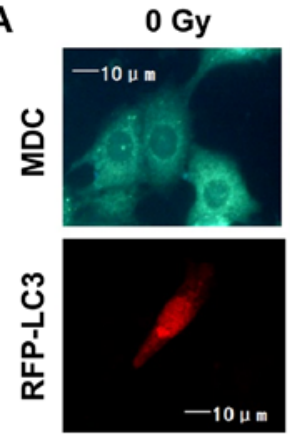

C

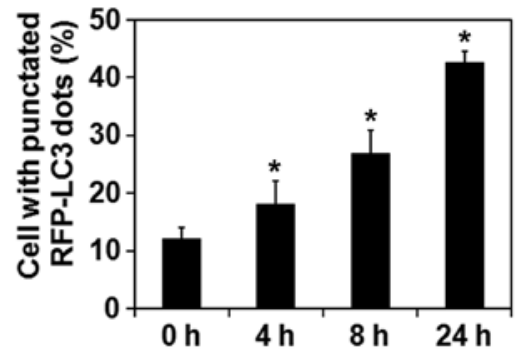

E

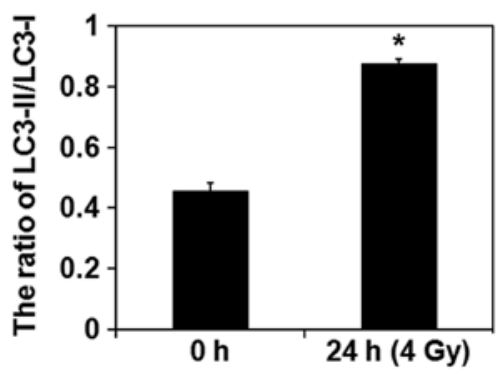

B

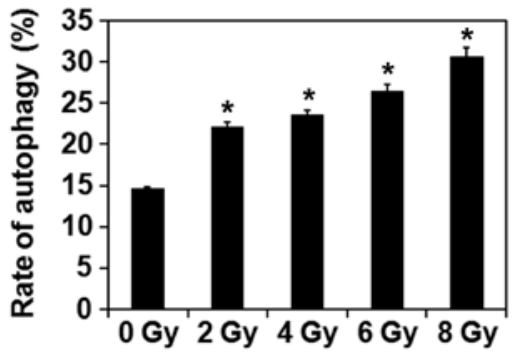

D

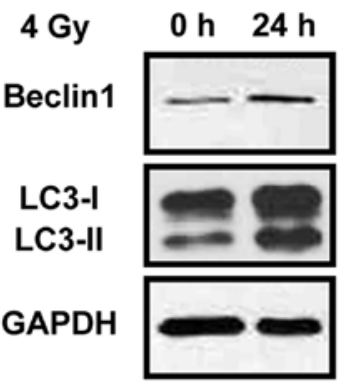

$\mathbf{F}$

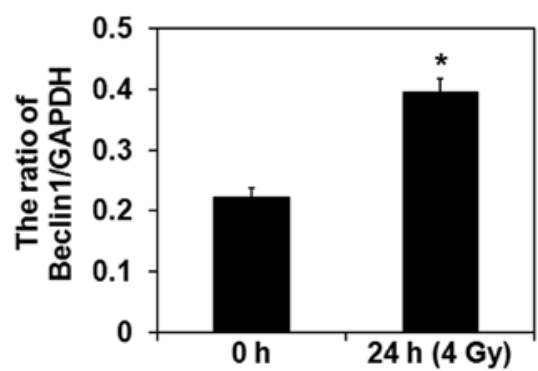

Figure 1. Radiation induces autophagy in MCF-7 breast cancer cells. (A) MCF-7 cells were exposed to 0 or 4 Gy radiation and then were stained by MDC (shown in green) $24 \mathrm{~h}$ later. In parallel, a set of MCF-7 cells were transfected with RFP-LC3 and then were exposed to 0 and 4 Gy radiation. Twenty-four hours after irradiation, the intracellular distribution of RPF-LC3 was examined. Scale bars, $10 \mu \mathrm{m}$. (B) MCF-7 cells were exposed to the indicated doses of radiation. Twenty-four hours later, the cells were stained with MDC and the percentage of positive cells were determined by flow cytometric analysis. (C) The percentage of cells exhibiting LC3 puncta was calculated at the indicated time points following irradiation. (D) Expression levels of Beclin 1, LC3I, LC3II, and GAPDH were examined by western blot analysis. " $\mathrm{P}<0.05$. The protein expression levels of LC3II relative to LC3I (E) and Beclin 1 relative to GAPDH (F) were each quantified from three independent experiments \pm SD. ${ }^{*} \mathrm{P}<0.05$.

radiation (Fig. 1D-F). Taken together, these results suggest that radiation induces autophagy in MCF-7 cells.

Radiation induces autophagic cell death in MCF-7 cells. Trypan blue dye exclusion assays were performed to detect cell death following the exposure of MCF-7 cells to 4-Gy radiation. As shown in Fig. 2A, the percentage of cells undergoing cell death increased in a time-dependent manner following irradiation. Next, the effect of inhibiting autophagy prior to radiation on cell viability was examined. For this purpose, MCF-7 cells were treated with or without 3-MA, and cell viability was examined in CCK-8 assays following the exposure of MCF-7 cells to various doses of radiation. As shown in Fig. 2B, treatment with 3-MA reversed the reduction in cell viability that was observed for the cells that were exposed to 2 , 4 , or 6 Gy radiation alone $(\mathrm{P}<0.05)$. These results suggest that autophagy may be the predominant pathway that mediates cell death following irradiation. However, inhibition of autophagy did not prevent cell death following the exposure of MCF-7 cells to 8-Gy radiation $(\mathrm{P}>0.05)$ (Fig. 2B). MDC staining further indicated that treatment with 3-MA significantly reduced the induction of autophagy in MCF-7 cells exposed to $4-\mathrm{Gy}$ radiation $(\mathrm{P}<0.05)$ (Fig. $2 \mathrm{C})$. However, there was no significant difference in the levels of apoptosis detected for the cells that were irradiated with or without 3-MA pretreatment $(\mathrm{P}>0.05)$ (Fig. 2D). When the autophagy-related genes, Atg5 and Beclin 1, were each knocked down in MCF-7 cells, both sets of cells were protected against cell death following exposure to 4-Gy radiation (Fig. 2E and F). In combination, these data indicate that radiation can lead to autophagic cell death in MCF-7 cells.

Gene microarray analysis of irradiated MCF-7 cells. To identify regulatory genes that participate in radiation-induced autophagic cell death, MCF-7 cells were exposed to 0,4 , or 8 Gy radiation and then were subjected to a gene microarray analysis $4 \mathrm{~h}$ later. Compared with the non-irradiated control cells, there were 160 differentially expressed genes that were detected after the exposure of the cells to 4 Gy radiation, including 93 genes that were upregulated (indicated in red in the row of $4 \mathrm{~Gy} 4 \mathrm{~h}$ ) and 67 genes that were downregulated (indicated in green in the row of 4 Gy 4 h) (Fig. 3). Overall, 
A

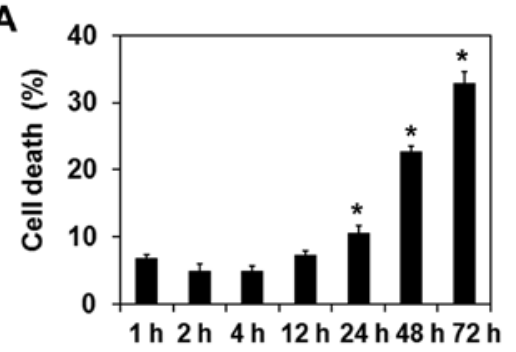

C
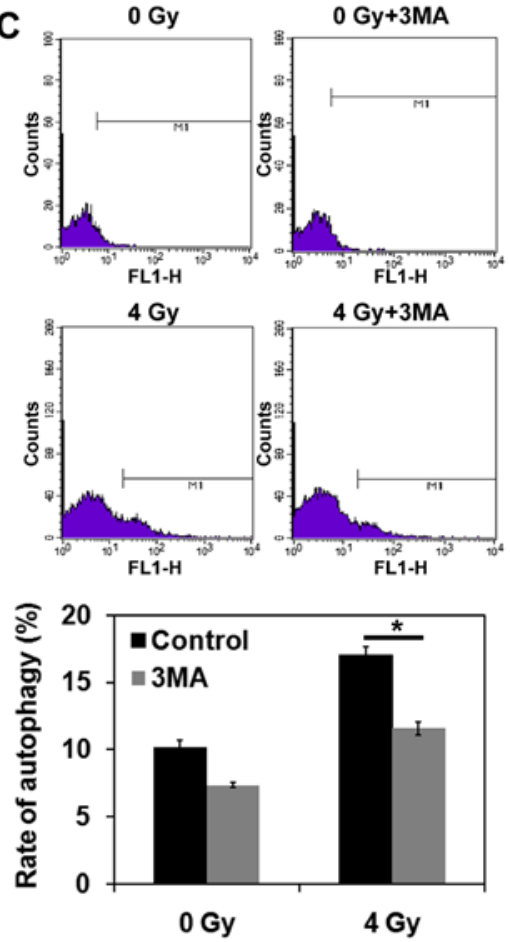

E

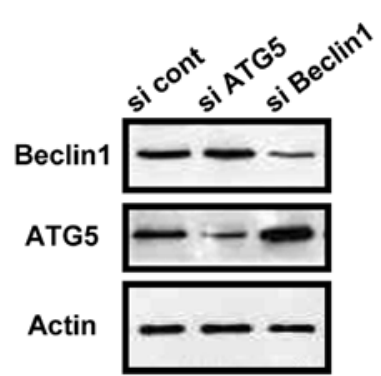

B

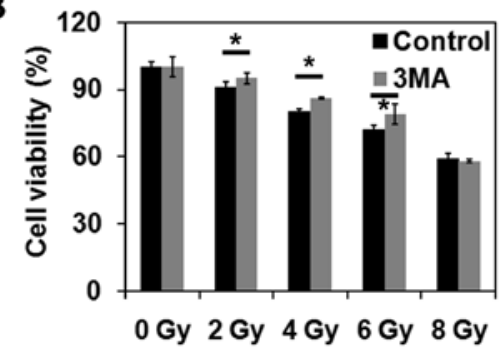

D
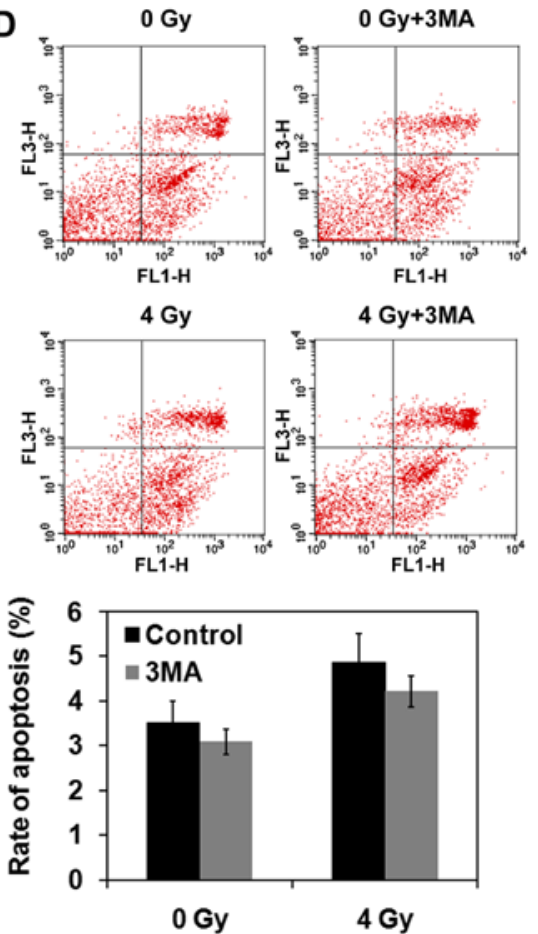

F

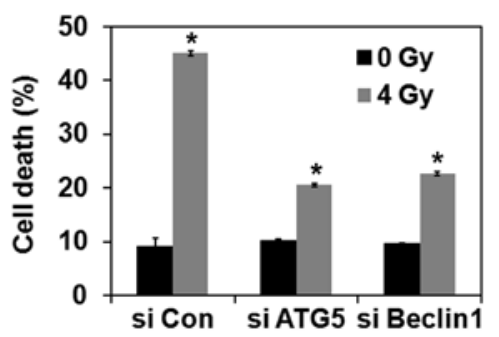

Figure 2. Radiation-induced autophagic cell death in MCF-7 cells. (A) MCF-7 cells were exposed to 4-Gy radiation. At the indicated time-points, cells were collected and assayed for trypan blue dye exclusion. The percentage of cells undergoing death was quantified by flow cytometry. (B) Cells were pre-treated with or without $2 \mathrm{mM} 3-\mathrm{MA}$ for $1 \mathrm{~h}$ before the cells were exposed to different doses of radiation. Twenty-four hours later, cell viability was determined with CCK-8 assays. (C) MCF-7 cells were pre-treated with or without $2 \mathrm{mM} 3$-MA for $1 \mathrm{~h}$ before the cells were exposed to 0 or 4 Gy radiation. Cells were stained with MDC to detect autophagic vacuoles and the percentage of positive cells was analyzed by flow cytometry. Quantitation of the rate of autophagy is shown in the bar graph to the right. (D) MCF-7 cells were subjected to Annexin V-FITC/PI double staining to detect cell apoptosis. Quantitation of these data \pm SD are presented in the bar graph to the right. (E) Western blot analysis following the introduction of siRNA targeting Atg 5 and Beclin 1 (siAtg5 and siBeclin1, respectively) vs. a control siRNA (sicon), in MCF-7 cells. Detection of actin was performed as a loading control. (F) Percentage of cell death was determined by trypan blue staining following the introduction of siRNA targeting Atg 5 and Beclin 1, vs. an siRNA control, 24 h after the cells were exposed to 0 or 4 Gy radiation. Data are presented as the mean $\pm \mathrm{SD}$ from three independent experiments. ${ }^{*} \mathrm{P}<0.05$.

these differentially expressed genes were associated with 10 signaling pathways. For the MCF-7 cells that were exposed to 8 Gy of radiation, a total of 236 differentially expressed genes were detected [including 121 upregulated genes (indicated in red in the row of 8 Gy $4 \mathrm{~h}$ ) and 115 downregulated genes (indicated in green in the row of 8 Gy $4 \mathrm{~h}$ )], and these were associated with 9 signaling pathways (Fig. 3). Furthermore, significant alterations in the expression of the autophagy- related genes, DRAM and TIGAR (also known as C12orf5), were detected following the exposure of the cells to 8-Gy radiation. Therefore, it appears that DRAM and TIGAR contribute to radiation-induced autophagic cell death in MCF-7 cells.

Characterization of DRAM and TIGAR in radiation-induced autophagic cell death in MCF-7 cells. In the qPCR analysis, mRNA levels of DRAM and TIGAR were found to be 


\begin{tabular}{|c|c|c|}
\hline SYMBOL & DRAM & C12orf5 \\
\hline Control.AVG & 201.6308 & 351.6373 \\
\hline 4Gy4h.AVG & 591.8154 & 1112.531 \\
\hline 4GY4h.Diff & 58.13822 & 84.53291 \\
\hline 8Gy4h.AVG & 586.8987 & 1268.673 \\
\hline 8Gy4h.Diff & 64.61238 & 78.52057 \\
\hline SEARCH_KEY & ILMN_20048 & ILMN_11001 \\
\hline ILMN_GENE & DRAM & C12ORF5 \\
\hline CHROMOSOME & 12 & 12 \\
\hline DEFINITION & $\begin{array}{l}\text { Homo } \\
\text { sapiens } \\
\text { damage- } \\
\text { regulated } \\
\text { autophagy } \\
\text { modulator } \\
\text { (DRAM), } \\
\text { mRNA. }\end{array}$ & $\begin{array}{c}\text { Homo } \\
\text { sapiens } \\
\text { chromosome } \\
12 \text { open } \\
\text { reading } \\
\text { frame } 5 \\
\text { (C12orf5), } \\
\text { mRNA. }\end{array}$ \\
\hline SYNONYMS & FLJ11259 & TIGAR \\
\hline TargetID & DRAM & C12ORF5 \\
\hline
\end{tabular}

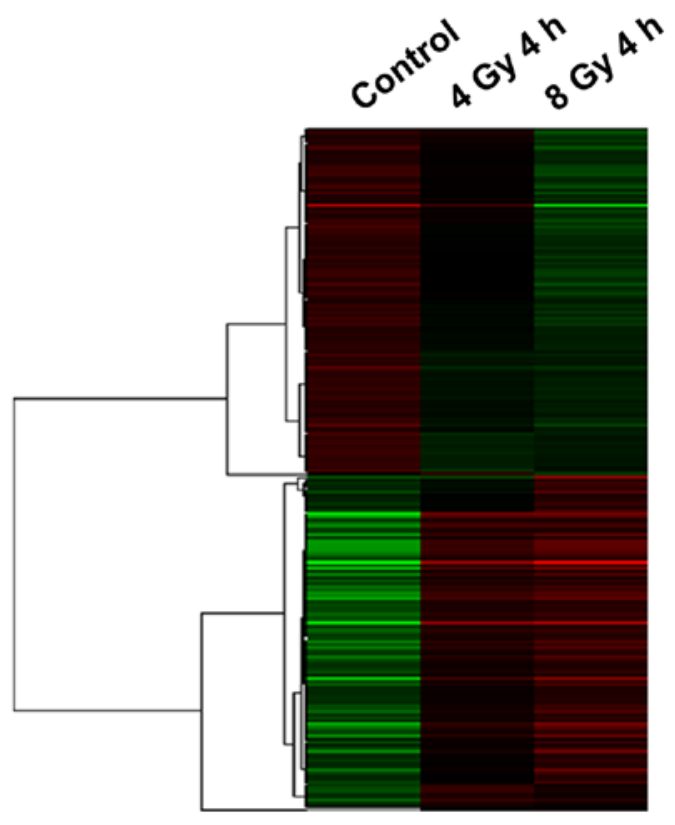

Figure 3. Gene expression data for genes that exhibited differential expression in irradiated vs. non-irradiated MCF-7 cells. Cells were exposed to 0,4 , or 8 Gy of radiation and $4 \mathrm{~h}$ later, each set of cells was subjected to a gene microarray analysis. The signals for genes that exhibited differential expression among the three sets of cell samples are shown. Signals shown in green represent upregulated gene expression and signals shown in red represent downregulated gene expression.

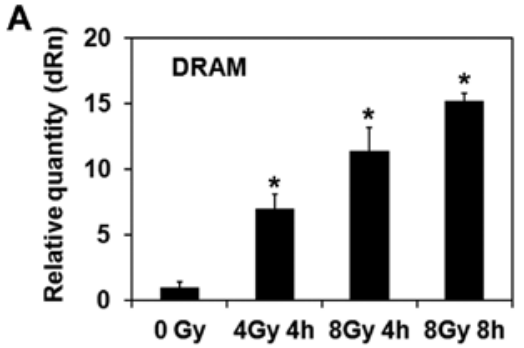

B

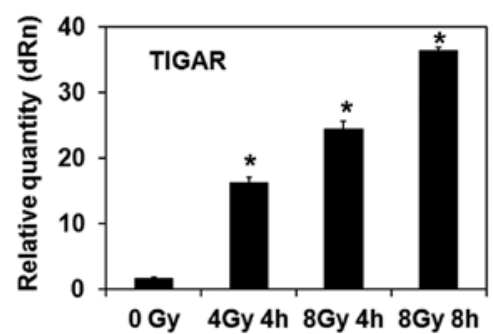

C

D
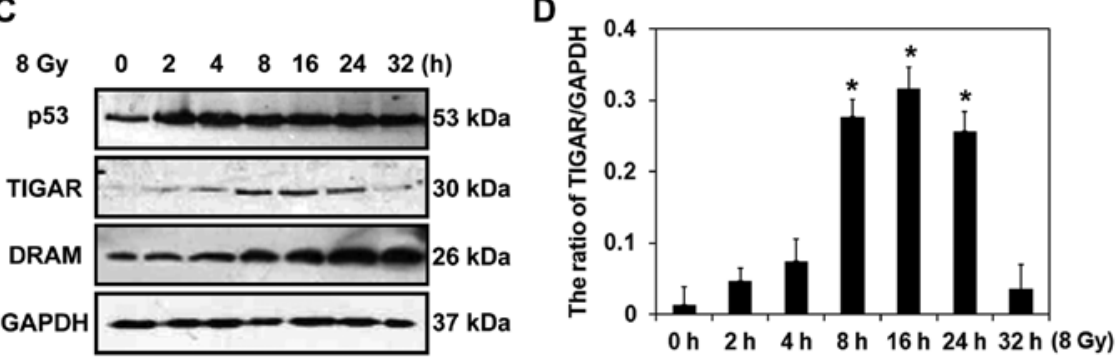

E

$\mathbf{F}$
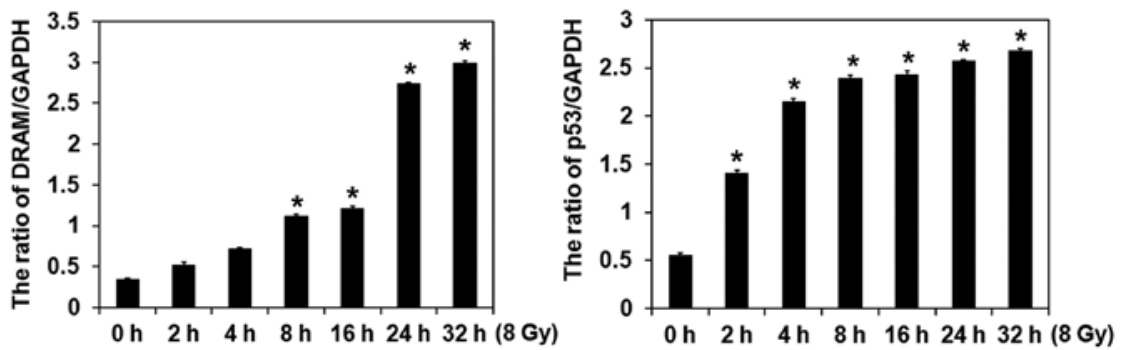

Figure 4. Increased DRAM and TIGAR expression detected in MCF-7 cells after irradiation. MCF-7 cells were exposed to 0,4 , or 8 Gy of radiation. At 4 or $8 \mathrm{~h}$ after irradiation, mRNA levels of (A) DRAM and (B) TIGAR were examined by qPCR. (C) MCF-7 cells were exposed to 8-Gy radiation and expression levels of p53, DRAM, TIGAR, and GAPDH were determined by western blot analysis at the indicated time-points. (D) Protein expression ratios of TIGAR relative to GAPDH were quantified from three independent experiments $\pm \mathrm{SD}$. ${ }^{*} \mathrm{P}<0.05$. The protein expression of DRAM (E) and p53 relative to GAPDH (F) were each quantified from three independent experiments $\pm \mathrm{SD}$. "P<0.05. 
A

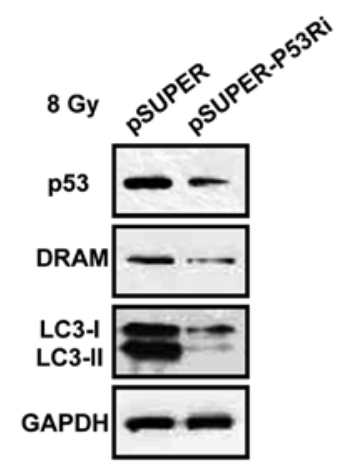

C

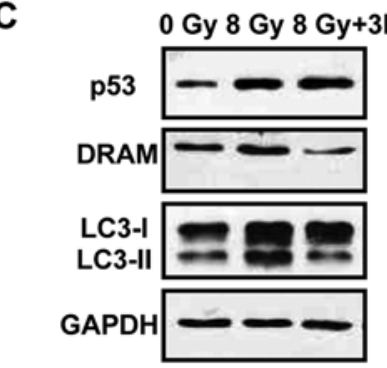

E

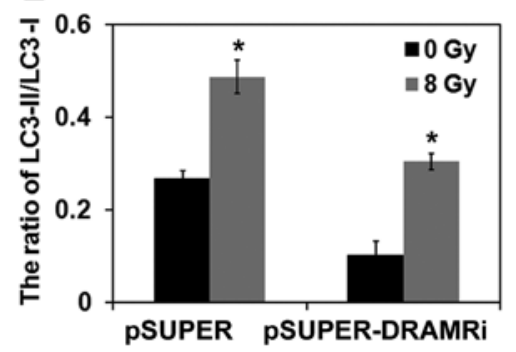

G

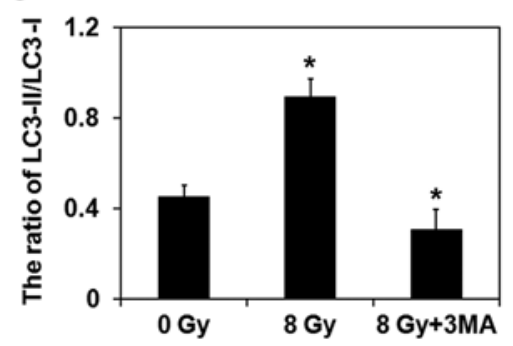

B
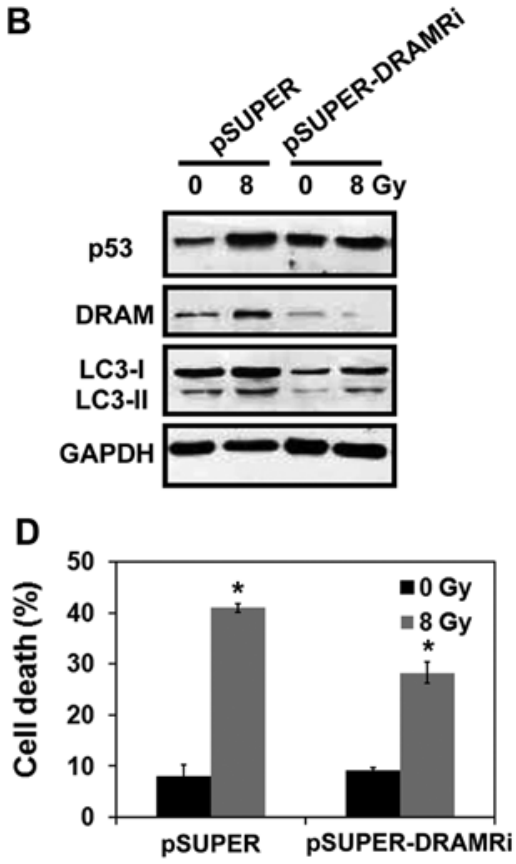

F

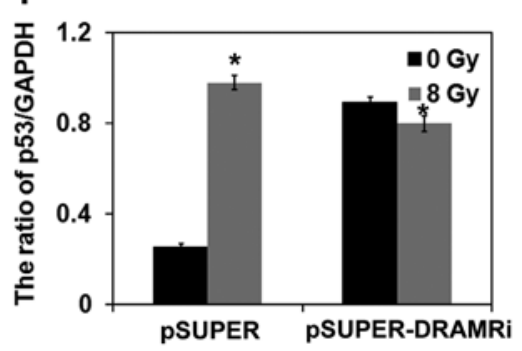

H

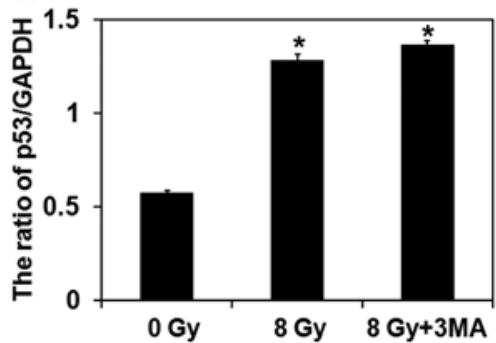

Figure 5. p53/DRAM regulates radiation-induced autophagic cell death in MCF-7 cells. (A) Expression levels of LC3-I, LC3-II, p53, and DRAM were analyzed by western blot analysis $24 \mathrm{~h}$ after control and p53-deficient MCF-7 cells were exposed to 8-Gy radiation. Detection of GAPDH was performed as a loading control. (B) Expression levels of LC3-I, LC3-II, p53, and DRAM were analyzed by western blot analysis $24 \mathrm{~h}$ after control and DRAM-deficient MCF-7 cells were exposed to 0 or 8 Gy radiation. Detection of GAPDH was performed as a loading control. (C) Expression levels of LC3 (including LC3-I and LC3-II), DRAM, and p53 were detected in MCF-7 cells that were exposed to 0 or 8 Gy radiation, as well as pretreatment with 3-MA prior to 8 Gy radiation. Detection of GAPDH was performed as a loading control. (D) Cell death was determined for MCF-7 cells that were transfected with control or DRAM-targeted shRNAs (pSUPER or pSUPER-DRAMRi) by trypan blue dye exclusion assays analyzed by flow cytometry. "P<0.05. DRAM-silenced MCF-7 cells were exposed to 0 or 8 Gy radiation and then the protein expression of LC3II relative to LC3I (E) and p53 relative to GAPDH (F) were each quantified from three independent experiments $\pm \mathrm{SD}$. ${ }^{*} \mathrm{P}<0.05$. MCF-7 cells were exposed to 0 or $8 \mathrm{~Gy}$ radiation in the presence or absence of 3MA. The protein expression of LC3II relative to LC3I (G) and p53 relative to GAPDH (H) were each quantified from three independent experiments \pm SD. ${ }^{*} \mathrm{P}<0.05$.

significantly elevated following the exposure of MCF-7 cells to radiation (Fig. 4A and B). As shown by western blot results, the levels of p53 gradually increased, as did the levels of DRAM, with time following radiation (Fig. 4C and E). However, while the expression of TIGAR gradually increased and peaked $16 \mathrm{~h}$ after the irradiation treatment, the levels decreased thereafter (Fig. 4C and D). Previously, DRAM was identified as a crucial effector of p53-activated autophagy. However, more recently, the lysosomal protein, DRAM has also been found to be required for p53-induced autophagy and cell death (20). Consistent with the DRAM data, expression levels of p53 were higher after the MCF-7 cells were exposed to radiation compared with the control cells (Fig. 4C and F). These data suggest that regulation of DRAM by p53 may mediate autophagy in MCF-7 cells following exposure to radiation.

p53/DRAM mediates radiation-induced autophagic cell death. To confirm the potential regulatory role of 
the p53/DRAM signaling pathway in radiation-induced autophagic cell death, a stable $p 53$-deficient MCF-7 cell line and DRAM-deficient MCF-7 cell line were established following the infection of MCF-7 cells with a pSUPERDRAM shRNA and a pSUPER-p53 shRNA. Western blot analysis confirmed that silencing of $\mathrm{p} 53$ and $D R A M$ were achieved (Fig. 5A and B). This in vitro model was then exposed to 8-Gy radiation. We found that radiation induced downregulation of DRAM and LC3-II in the p53-deficient MCF-7 cells (Fig. 5A). This in vitro model was then exposed to 8-Gy radiation, and radiation-induced upregulation of $\mathrm{p} 53$, DRAM, and LC3-II were reversed compared with wild-type MCF-7 cells that were exposed to the same radiation in a $D R A M$-deficient MCF-7 cell line (Fig. 5B). These results are in agreement with the results of the statistical analysis in the graph shown in Fig. 5E and F). Previously, it was reported that siRNA-mediated knockdown of p53 was able to completely block DRAM expression and significantly reduce the level of autophagy (11). However, in the present study, silencing of DRAM only partially blocked autophagy, which might be due to the upregulated p 53 level after irradiation. It is also possible as p53 may induce autophagy by mediating other autophagy related genes. As shown in Fig. 5C, inhibition of autophagy by 3-MA further reduced the radiation-induced upregulation of LC3-II as well as DRAM, while no significant difference was detected in the expression of p53 in the wild-type MCF-7 cells that were irradiated (Fig. 5G and H). In trypan blue exclusion assays, DRAM-deficient MCF-7 cells also exhibited a marked reduction in cell death rate after irradiation (Fig. 5D). Thus, the present findings suggest that the p53/DRAM signaling pathway mediates radiation-induced autophagic cell death in MCF-7 cells.

\section{Discussion}

Ionizing radiation is an efficient adjuvant therapy for the management of breast cancer after surgery as it reduces local recurrence and has been shown to prolong the long-term survival of patients (21). However, breast cancer cells can develop radioresistance, and the ability to promote radiosensitivity could potentially improve the therapeutic outcome in these cases (22). In recent studies, autophagy has been shown to play a key role in the radiosensitivity and radioresistance of various cancer therapies (6). However, the precise role of autophagy in radiation-induced cytotoxicity of breast cancer cells has not been fully characterized. In the present study, radiation triggered autophagic cell death in MCF-7 breast cancer cells, and we provide evidence to indicate this process is modulated by the p53/DRAM signaling pathway.

Increased autophagic activity in irradiated MCF-7 cells was demonstrated with the presence of enhanced MDC staining, an accumulation of LC3-positive puncta, and upregulated expression of autophagy-related genes. Moreover, these observations were accompanied by enhanced cytotoxicity, suggesting the involvement of autophagy in radiation-induced cell injury. Similarly, enhanced autophagy has been observed in other studies of breast cancer cells that were subjected to ionizing radiation $(14,23)$. However, it should be noted that autophagy can act as a double-edged sword in modulating radiation-triggered cancer cell death (24). For example, Han et al reported that blockage of autophagy overcame the radioresistance of breast cancer cells (25), and Bristol et al reported that an inhibition of autophagic activity in MCF-7 cells aggravated cytotoxicity and a cytoprotective role was indicated for autophagy induced by radiation (26). Thus, it appears that autophagy may favor cancer cell survival and radioadaption by contributing to the maintenance of intracellular homeostasis in cells (27). However, radiation has also been shown to induce autophagic cell death in MCF-7 cells (28). In accordance with this finding, the results of the present study demonstrate that autophagy can act as a pro-death mechanism. Specifically, it was demonstrated in the present study that inhibition of the autophagic pathway by pharmacological interference or with the knockdown of autophagy-related genes could prevent cell death-induced radiation. Therefore, it is hypothesized that augmentation of autophagy may sensitize breast cancer cells to radiotoxicity.

In the gene microarray analysis that was performed, marked changes in expression of the autophagy-related genes, $D R A M$ and TIGAR, were observed. DRAM plays a crucial role in mediating a cell's response to DNA damage (9), and is also a downstream target of p53. Furthermore, activation of the p53/DRAM signaling pathway has been found to mediate the induction of autophagy in breast cancer cells by anticancer reagents such as doxorubicin (29) and acetazolamide (13). Consistent with these findings, overexpression of $D R A M$ and $p 53$ were detected following the irradiation of MCF-7 breast cancer cells in the present study. Conversely, silencing of $p 53$ decreased DRAM and LC3-II. Silencing of $D R A M$ reversed radiation-induced of the autophagy-related gene, $L C 3-I I$ and increased the expression levels of p53. The latter observation may involve a compensatory mechanism by which radiation-induced upregulation of p53 is reversed, and thus, a positive feedback loop may exist between $\mathrm{p} 53$ and DRAM. It has been reported that DRAM-induced autophagy was also accompanied by an increased number of autophagic vacuoles and upregulated expression of LC3 (10). Knockdown of DRAM attenuates the ability of wild-type p53 to induce autophagy (30). Furthermore, knockdown of DRAM in MDA-MB-231 cells, which represent a p53-mutated breast cancer cell line, did not induce DRAM-1 expression or autophagy (29). It was also observed in the present study that DRAM silencing partially prevented radiation-induced cell death in MCF-7 cells, and this result indicates a potential role for the p53/DRAM-autophagy axis in radiation-induced cytotoxicity of breast cancer cells.

In conclusion, the results of the present study demonstrate that ionizing radiation induces autophagic cell death of MCF-7 cells via the p53/DRAM signaling pathway, and they also identify potential targets for improving radiosensitivity in the treatment of breast cancer.

\section{References}

1. Galluzzi L, Vicencio JM, Kepp O, Tasdemir E, Maiuri MC and Kroemer G: To die or not to die: That is the autophagic question. Curr Mol Med 8: 78-91, 2008.

2. Xie Z and Klionsky DJ: Autophagosome formation: Core machinery and adaptations. Nat Cell Biol 9: 1102-1109, 2007.

3. Dalby KN, Tekedereli I, Lopez-Berestein G and Ozpolat B: Targeting the prodeath and prosurvival functions of autophagy as novel therapeutic strategies in cancer. Autophagy 6: 322-329, 2010. 
4. Fitzmaurice C, Dicker D, Pain A, Hamavid H, Moradi-Lakeh M, MacIntyre MF, Allen C, Hansen G, Woodbrook R, Wolfe C, et al; Global Burden of Disease Cancer Collaboration: The Global Burden of Cancer 2013. JAMA Oncol 1: 505-527, 2015.

5. Tsoutsou PG, Koukourakis MI, Azria D and Belkacémi Y: Optimal timing for adjuvant radiation therapy in breast cancer: A comprehensive review and perspectives. Crit Rev Oncol Hematol 71: 102-116, 2009.

6. Yang Y, Yang Y, Yang X, Zhu H, Guo Q, Chen X, Zhang H, Cheng $\mathrm{H}$ and Sun $\mathrm{X}$ : Autophagy and its function in radiosensitivity. Tumour Biol 36: 4079-4087, 2015.

7. Gewirtz DA: The four faces of autophagy: Implications for cancer therapy. Cancer Res 74: 647-651, 2014.

8. Juenemann K and Reits EA: Alternative macroautophagic pathways. Int J Cell Biol 2012: 189794, 2012

9. Crighton D, Wilkinson S, O'Prey J, Syed N, Smith P, Harrison PR, Gasco M, Garrone O, Crook T and Ryan KM: DRAM, a p53-induced modulator of autophagy, is critical for apoptosis Cell 126: 121-134, 2006.

10. Zhu BS, Zhao K, Jia X, Wu YY and Xing CG: Effects of damage-regulated autophagy regulator gene on the SGC7901 human gastric cancer cell line. Oncol Lett 8: 657-662, 2014.

11. Liu K, Shi Y, Guo XH, Ouyang YB, Wang SS, Liu DJ, Wang AN, Li $\mathrm{N}$ and Chen DX: Phosphorylated AKT inhibits the apoptosis induced by DRAM-mediated mitophagy in hepatocellular carcinoma by preventing the translocation of DRAM to mitochondria. Cell Death Dis 5: e1078, 2014.

12. Kim TH, Kim HS, Kang YJ, Yoon S, Lee J, Choi WS, Jung JH and Kim HS: Psammaplin A induces Sirtuin 1-dependent autophagic cell death in doxorubicin-resistant MCF-7/adr human breast cancer cells and xenografts. Biochim Biophys Acta 1850 401-410, 2015.

13. Mohammadpour R, Safarian S, Ejeian F, Sheikholya-Lavasani Z, Abdolmohammadi $\mathrm{MH}$ and Sheinabi N: Acetazolamide triggers death inducing autophagy in T-47D breast cancer cells. Cell Biol Int 38: 228-238, 2014.

14. Yi H, Liang B, Jia J, Liang N, Xu H, Ju G, Ma S and Liu X: Differential roles of miR-199a-5p in radiation-induced autophagy in breast cancer cells. FEBS Lett 587: 436-443, 2013.

15. Liang N, Jia L, Liu Y, Liang B, Kong D, Yan M, Ma S and Liu X: ATM pathway is essential for ionizing radiation-induced autophagy. Cell Signal 25: 2530-2539, 2013.

16. Yang C, Tang X, Guo X, Niikura Y, Kitagawa K, Cui K, Wong ST, Fu L and Xu B: Aurora-B mediated ATM serine 1403 phosphorylation is required for mitotic ATM activation and the spindle checkpoint. Mol Cell 44: 597-608, 2011.
17. Livak KJ and Schmittgen TD: Analysis of relative gene expression data using real-time quantitative PCR and the 2(-delta delta C(T)) method. Methods 25: 402-408, 2001.

18. Vázquez CL and Colombo MI: Assays to assess autophagy induction and fusion of autophagic vacuoles with a degradative compartment, using monodansylcadaverine (MDC) and DQ-BSA. Methods Enzymol 452: 85-95, 2009.

19. Kimura S, Noda T and Yoshimori T: Dissection of the autophagosome maturation process by a novel reporter protein, tandem fluorescent-tagged LC3. Autophagy 3: 452-460, 2007.

20. Crighton D, Wilkinson S and Ryan KM: DRAM links autophagy to p53 and programmed cell death. Autophagy 3: 72-74, 2007.

21. Lee LJ and Harris JR: Innovations in radiation therapy (RT) for breast cancer. Breast 18 (Suppl 3): S103-S111, 2009.

22. Kaidar-Person O, Lai C, Kuten A and Belkacemi Y; AROME 'The Infinite Maze' of breast cancer, signaling pathways and radioresistance. Breast 22: 411-418, 2013.

23. Chaachouay H, Ohneseit P, Toulany M, Kehlbach R, Multhoff G and Rodemann HP: Autophagy contributes to resistance of tumor cells to ionizing radiation. Radiother Oncol 99: 287-292, 2011.

24. Hönscheid P, Datta K and Muders MH: Autophagy: Detection, regulation and its role in cancer and therapy response. Int $\mathrm{J}$ Radiat Biol 90: 628-635, 2014.

25. Han MW, Lee JC, Choi JY, Kim GC, Chang HW, Nam HY, Kim SW and Kim SY: Autophagy inhibition can overcome radioresistance in breast cancer cells through suppression of TAK1 activation. Anticancer Res 34: 1449-1455, 2014.

26. Bristol ML, Di X, Beckman MJ, Wilson EN, Henderson SC, Maiti A, Fan Z and Gewirtz DA: Dual functions of autophagy in the response of breast tumor cells to radiation: Cytoprotective autophagy with radiation alone and cytotoxic autophagy in radiosensitization by vitamin D 3. Autophagy 8: 739-753, 2012.

27. Szumiel I: Radiation hormesis: Autophagy and other cellular mechanisms. Int J Radiat Biol 88: 619-628, 2012.

28. Zhong R, Xu H, Chen G, Zhao G, Gao Y, Liu X, Ma S and Dong L: The role of hypoxia-inducible factor- $1 \alpha$ in radiation-induced autophagic cell death in breast cancer cells. Tumour Biol 36: 7077-7083, 2015.

29. Gomes LR, Vessoni AT and Menck CF: Three-dimensional microenvironment confers enhanced sensitivity to doxorubicin by reducing p53-dependent induction of autophagy. Oncogene 34: 5329-5340, 2015.

30. Takahashi M, Kakudo Y, Takahashi S, Sakamoto Y, Kato S and Ishioka C: Overexpression of DRAM enhances p53-dependent apoptosis. Cancer Med 2: 1-10, 2013. 